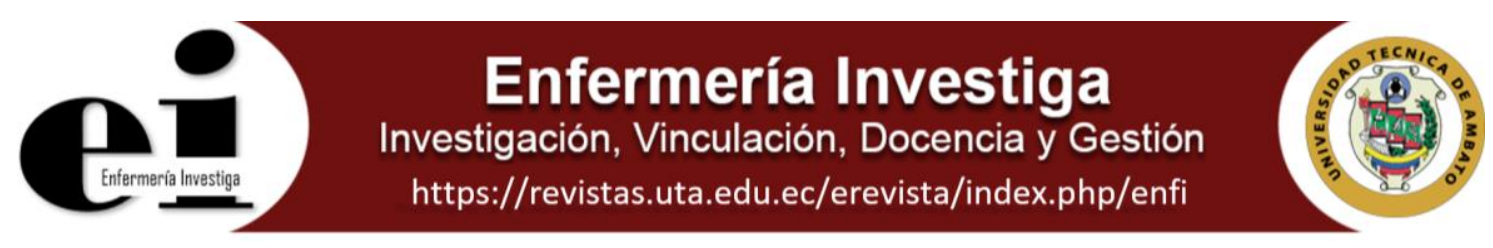

\title{
INTERVENCIÓN DE ENFERMERÍA PARA EL BIENESTAR HOLÍSTICO DEL ADULTO MAYOR
}

\section{NURSING INTERVENTION FOR THE HOLISTIC WELL-BEING OF THE OLDER ADULT}

Verónica Gavilanes ${ }^{1,2}$ https://orcid.org/0000-0002-0530-2011, Eva Mendoza de Graterol2,3 https://orcid.org/0000-0002-41909160, Blanca Costales ${ }^{4}$ https://orcid.org/0000-0001-9728-238X

${ }^{1}$ Hospital General Ambato, Ecuador.

2Universidad de Zulia, División de Estudios para Graduados, Doctorado de Ciencias de la Salud, Venezuela

3Universidad de Zulia, Escuela de Enfermería, Facultad de Medicina, Venezuela

${ }^{4}$ Centro de Salud Tipo C Latacunga, Ecuador.

2477-9172 / 2550-6692 Derechos Reservados (c) 2021 Universidad Técnica de Ambato, Carrera de Enfermería. Este es un artículo de acceso abierto distribuido bajo los términos de la Licencia Creative Commons, que permite uso ilimitado, distribución y reproducción en cualquier medio, siempre que la obra original es debidamente citada

Recibido: 23 de octubre 2020

Aceptado: 23 de diciembre 2020

\section{Resumen:}

Introducción: el envejecimiento implica alteraciones funcionales, cognitivas psicológicas y sociales que disminuyen la calidad de vida de los adultos mayores. Objetivo: determinar el efecto de la intervención de enfermería para el bienestar holístico del adulto mayor. Método: investigación cuantitativa, diseño cuasi-experimental de un sólo grupo (150 adultos mayores), con pretest-postest utilizando tres instrumentos: Barthel, Zimet y el test de Minimental, intervención de enfermería con estrategias de autocuidado de actividad física y terapia de reminiscencia, durante 12 meses, datos procesados con el software estadístico (SPSS Estatistics 24.0 para windows), por medio de distribución porcentual y la prueba de $\mathrm{T}$ de Student con una significancia estadística $(p<0.05)$. Resultados: predominó la edad de 60-64 años $(36,7 \%)$, género masculino $62,7 \%$, ubicación en el Instituto Geriátrico Sagrado Corazón 53,4\%, después de la intervención de enfermería se incrementó la percepción de apoyo social, la independencia de las actividades básicas de la vida diaria para la alimentación, deambular, subir y bajar escaleras, hábitos fisiológicos e higiénicos, en el estado cognitivo se evidenció disminución del deterioro cognitivo y aumento de la evaluación borderline y normal, con una diferencia significativa $p<0,001$ por medio de la escala de Zimet, índice de Barthel y el test de Minimental con las estrategias de autocuidado de actividad física y terapia de reminiscencia. Conclusiones: se obtuvo una elevación de la percepción de apoyo social, la independencia para realizar las actividades básicas de la vida diaria y aumento del estado cognitivo, que incrementaron el bienestar y la calidad de vida de los adultos mayores

Palabras claves: adulto mayor, estrategias de enfermería, escala de Zimet, índice de Barthel y el test de Minimental

\section{Abstract:}

Introduction: Aging implies functional, cognitive, psychological and social alterations that decrease the quality of life of older adults. Objective: To determine the effect of the nursing intervention for the holistic well-being of the elderly. Method: Quantitative investigation, quasiexperimental design of a single group (150 older adults), with pretest-posttest using three tools: Barthel, Zimet and the Minimental test, nursing intervention with self-care strategies of physical activity and reminiscence therapy, for 12 months, data processed with statistical software (SPSS Statistics 24.0 for Windows), by means of percentage distribution and Student's T-Test with statistical significance $(p<0.05)$. Results: the age of $60-64$ years predominated (36.7\%), male gender $62.7 \%$, location in Instituto Geriátrico Sagrado Corazón 53.4\%, after the nursing intervention, the perception of social support increased, the independence of basic activities of daily life for eating, walking, going up and down stairs, physiological and hygienic habits, in the cognitive state a decrease in cognitive deterioration was evidenced and an increase in borderline and normal evaluation, with a significant difference $p<0.001$ using the Zimet scale, Barthel's index and the Minimental test with the self-care strategies of physical activity and reminiscence therapy. Conclusions: an increase of the perception of social support, independence to carry out the basic activities of daily life and an increase in cognitive status was obtained, which increased the well-being and quality of life of the elderly.

Keywords: elderly, nursing strategies, Zimet scale, Barthel index and the Minimental test 


\section{INTRODUCCIÓN:}

El envejecimiento es un proceso universal, continuo, dinámico, progresivo, declinante y heterogéneo, en el que ocurren cambios biopsicosociales resultantes de la interacción de factores genéticos, sociales, culturales, del estilo de vida y la presencia de enfermedades (1), es decir, una insuficiencia para mantener la homeostasis, asociada con una disminución en la vitalidad y un incremento en la vulnerabilidad del individuo $(2,3)$, se debe tomar en cuenta en los adultos mayores la fragilidad sensorial para mejorar el estado de salud, que requiere un diagnóstico precoz con una rehabilitación oportuna $(4,5-6)$.

Actualmente la mayoría de las personas aspiran vivir más de 60 años, para el año 2050 la proporción de los adultos mayores a nivel mundial se duplicará, pasando del $11 \%$ al $22 \%$ y en el transcurso de medio siglo de 605 millones a 2000 millones. En las últimas décadas, el mundo ha sufrido el fenómeno denominado "Encanecimiento Mundial", caracterizado por la inversión de la pirámide poblacional, debido al aumento en la esperanza de vida asociado a la implementación de programas geriátricos de rehabilitación para un bienestar holístico de los adultos mayores (7-8).

El incremento de la esperanza de vida y el descenso en las tasas de fecundidad, son las principales causas del rápido envejecimiento a nivel mundial, un niño nacido en Brasil en el año 2015 puede aspirar a vivir 20 años más que en el año 1965. En algunos países en el año 2015, tan solo un habitante de cada diez era mayor de 60 años, esta cifra ha aumentado a uno de cada tres, es decir, el ritmo de envejecimiento de la población es mucho más rápido que en el pasado (8), una vida más larga es un recurso extremadamente valioso, sin embargo, el alcance de las oportunidades de una mayor longevidad dependerá en gran medida de las fortalezas y debilidades en las que los adultos mayores puedan disfrutar de un bienestar holístico (9). Si las personas viven años adicionales en buen estado de salud, se adaptarán mejor a las actividades cotidianas y laborales (10), pero en la mayoría de los casos el aumento de la longevidad no viene acompañado de un período prolongado de salud, lamentablemente las personas mayores de la actualidad no tienen mejor salud que sus padres a la misma edad $(11,12)$.

El adulto mayor debe enfrentarse no sólo a cambios físicos e intelectuales propios de la edad, sino también a cambios sociales que conllevan a una disminución de las relaciones interpersonales y a un aislamiento social, ya que algunas personas deben ser manejadas en un lugar de cuidado y alejados de su entorno familiar (13), estas situaciones llevan a los adultos mayores a estar constantemente expuestos a factores estresantes del medio ambiente social, que requieren que el individuo reajuste sus patrones habituales de comportamiento $(14,15)$.

En geriatría las intervenciones en Enfermería permiten la prevención, el diagnóstico precoz de enfermedades crónicas y promover el aprendizaje de conductas para fortalecer la capacidad funcional de los adultos mayores, a través del autocuidado, es decir, el desarrollo de aptitudes, habilidades y destrezas que permitan ayudar a mantener la independencia funcional, los procesos vitales y el funcionamiento integrado, para hacer menos grave la enfermedad o discapacidad (16), además el mantenimiento de su autovalencia, el fomento del ejercicio, la promoción de comportamientos y estilos de vida saludables (17-18), para evitar la declinación en las funciones intelectuales tales como análisis, síntesis, razonamiento aritmético, ingenio e imaginación, percepción y memoria visual, es importante hacer notar que mientras el adulto mayor se mantenga activo y productivo el deterioro de sus facultades intelectuales será menor (19-20), por esta razón el objetivo de la investigación es determinar el efecto de las estrategias de enfermería en el bienestar holístico de los adultos mayores

\section{MÉTODO}

Investigación cuantitativa con diseño cuasi experimental de un sólo grupo pretest-postest, en una muestra de 150 adultos mayores, cada uno con un familiar significativo y/o cuidador representando una muestra censal, dado que representa el $100 \%$ de los adultos registrados en dos Institutos de atención geriátrica: Centro de Atención Integral del Adulto Mayor y Sagrado Corazón de Jesús del Cantón Ambato, Ecuador, que mediante la intervención se aplicaron estrategias de enfermería para el cuidado directo, aplicando los siguientes instrumentos:

1. La Escala Multidimensional de Apoyo Social (Multidimensional Scale of Perceived Social Support (MSPSS) de Zimet, Dahlem, Zimet \& Farley: conformada por 12 afirmaciones (ítems) que evalúan la percepción de apoyo social. Estos ítems se agrupan en 3 factores, el apoyo social de pares (ítems 6, 7, 9 y 12), el apoyo de la familia (ítems 3, 4, 8 y 11) y la percepción de apoyo de la pareja u otras figuras significativas (ítems 1, 2, 5), la confiabilidad de la MSPSS a través del coeficiente alpha de Cronbach de 0.86 para la escala total, traducida y adaptada para su uso en Chile por Arechavala y Miranda, donde la principal adaptación realizada consistió en reducir la escala de frecuencia a 4 niveles, desde 1 que indica casi nunca, hasta 4 que señala casi siempre o siempre, así la escala va desde un puntaje mínimo de 12 a un máximo de 48, en donde a mayor puntaje se incrementa el apoyo social percibido $(21,22-23)$.

2. La escala de Barthel evalúa la independencia para realizar las actividades básicas de la vida diaria (ABVD), se le asignan puntos por cada actividad que realice de forma independiente o con algún grado de ayuda que precise, puntuándose de 0 a 100, valorando las actividades con 0 , 5,10 o 15 puntos, con categorización del resultado en diferentes grados de dependencia. en el cual las actividades a evaluar son las siguientes: capacidad de arreglarse, bañarse, alimentarse, ir al retrete, deambular, subir escaleras, control de esfínter anal y vesical, la fiabilidad estimada por el alfa de Cronbach, con un valor de 0,90 para la escala total. El rango de puntuación puede variar entre 0 (completamente dependiente) y 100 puntos (completamente independiente), 91-99: dependencia escasa, 61-90: dependencia moderada y 21-60: dependencia severa (24).

3. El test de minimental es la primera versión en castellano del MINI-MENTAL STATE EXAMINATION (MMSE), instrumento práctico para valorar el estado cognitivo es ampliamente utilizado por su rapidez y facilidad en la puntuación para la detección de deterioro cognitivo global, 30 preguntas dicotómico, las cuales exploran diversos dominios cognoscitivos. La puntuación tiene un rango de 0 a 1; dependiendo de la respuesta, ésta se califica con 0 si la respuesta es incorrecta y con 1 si es correcta. Los ítems de esta escala se dividen en 5 funciones mentales superiores:
a. Orientación en tiempo y lugar
b. Memoria a corto plazo
c. Atención y cálculo 

d. Memoria diferida
e. Lenguaje oral y escrito.

Para su calificación se realiza la suma total de cada apartado correspondiente a la función mental superior explorada, el punto de corte es 24; si el resultado total es igual o menor a 23 es probable que la persona presente deterioro cognoscitivo, con una fiabilidad estimada por el alfa de Cronbach del 0,90 para la escala total (25).

Criterios de Inclusión son las personas de los grupos etarios correspondientes al adulto mayor según los criterios de la OMS, entre la edad de 60 a 70 años, sin distingo de raza o género, con capacidad cognitiva y atendidos en los centros de atención del cantón Ambato, en el periodo comprendido desde mayo de 2018 a mayo de 2019, y que acepten participar en el estudio mediante la firma del consentimiento informado de cada uno de ellos.

Criterios de exclusión: ancianos que estén en aislamiento, o con enfermedades neurodegenerativas.

\section{Intervenciones de Enfermería.}

Las intervenciones de Enfermería se basaron en estrategias de autocuidado para mejorar la capacidad funcional de los adultos mayores, realizadas en un periodo de 12 meses, entre el 01 de febrero 2018 al 01 de febrero 2019, incluyendo actividades básicas de la vida diaria: (comer, vestirse, aseo personal, caminar), actividades socio-recreativas, ejercicio físico aeróbico (caminar, subir escaleras, bailar, etc) e incremento de la capacidad cognoscitiva (26-27). Debido a las condiciones fisiológicas de los adultos mayores la intervención de enfermería de la actividad física se inició progresivamente de 10-15 minutos, dos o tres veces al día (al menos 30 minutos

diarios), luego se incrementó hasta una duración de 45-60 minutos (27), diariamente, para mejorar la fuerza, sincronización y fatiga muscular, con el objetivo de fortalecer los músculos de las articulaciones débiles, movilizar las articulaciones limitadas antes de practicar los ejercicios, evitando superficies duras y la hipotensión (28-29, 30-31).

La terapia de reminiscencia consiste en rememorar recuerdos de la historia personal del sujeto, estimular el proceso de la memoria, con la reactivación del pasado y el mantenimiento de su propia identidad y orientación autopsíquica, con grupos pequeños de 2 a 6 adultos mayores, con una duración máxima de las sesiones de 30-40 minutos, complementadas con actividades lúdicas y físicas de acuerdo a condición física y cognitiva de cada adulto mayor. Lactividades siguiendo los dos pasos indispensables, el primero, enseñar al paciente materiales y estímulos multisensoriales, como una fotografía o el relato de un suceso histórico, vinculados con su propia experiencia o con los acontecimientos experimentados por su generación y segundo, a partir de ese material, el paciente evocará sus recuerdos asociados y hará comentarios personales, de acuerdo a la caja de recuerdos (objetos personales: fotografías, música, ropas, documentación, diarios, libros), libro autobiográfico (familiar que conozca muy de cerca la vida del adulto mayor, para tener la historia de vida) y datos socio-históricos y/o culturales (información sobre sucesos históricos, sociales y culturales más importantes de la generación del adulto mayor) (32-33).

\section{RESULTADOS}

En las características demográficas en el grupo de adultos mayores: predomina la edad de $60-64$ años (36,7\%), seguido de 65-69 años (28,6\%), 70-74 años (24\%) y 75-79 años $(10,7 \%)$, el género masculino $94(62,7 \%)$ de los adultos mayores y femenino $56(37,3 \%)$, del Instituto Geriátrico Sagrado Corazón 90 53,4\%, Municipal 46,6\% (60) (tabla 1).

TABLA 1

CARACTERÍSTICAS DEMOGRÁFICAS DE LOS ADULTOS MAYORES

\begin{tabular}{|c|c|c|}
\hline $\begin{array}{l}\text { CARACTERISTICAS } \\
\text { DEMOGRÁFICAS }\end{array}$ & $\mathbf{N}^{\circ}$ & $\%$ \\
\hline \multicolumn{3}{|l|}{ EDADES } \\
\hline $60-64$ & 55 & 36,7 \\
\hline $65-69$ & 43 & 28,6 \\
\hline $70-74$ & 36 & 24 \\
\hline $75-79$ & 16 & 10,7 \\
\hline Total & 150 & 100 \\
\hline \multicolumn{3}{|l|}{ GÉNERO } \\
\hline Femenino & 56 & 37,3 \\
\hline Masculino & 94 & 62,7 \\
\hline Total & 150 & 100 \\
\hline \multicolumn{3}{|c|}{ INSTITUTO GERIÁTRICO } \\
\hline Municipal & 60 & 46,6 \\
\hline Sagrado Corazón & 90 & 53,4 \\
\hline Total & 150 & 100 \\
\hline
\end{tabular}

Fuente: Instrumento aplicado por los autores

En la distribución de los adultos mayores de acuerdo a la Escala Multidimensional de Apoyo Social de familiares y otros significativos antes de la intervención de enfermería 65 $(43,3 \%)$ con frecuencia perciben el apoyo de sus familiares y otros significativos, $38(25,4 \%)$ a veces lo perciben, 27 $(17,9 \%)$ casi nunca y $20(13,4 \%)$ casi siempre o siempre perciben apoyo familiar, después de la intervención de enfermería $77(51,4 \%)$ con frecuencia sienten apoyo de familiares, $34(22,7 \%)$ casi siempre o siempre perciben apoyo, $22(14,5 \%)$ a veces y el $17(11,4 \%)$ casi nunca lo perciben. Además, en el apoyo social de amigos, vecinos o compañeros de trabajo antes de la intervención de enfermería $63(41,9 \%)$ con frecuencia perciben el apoyo, 48 $(32,1 \%)$ a veces lo perciben, $23(15,3 \%)$ casi nunca y 16 
$(10,6 \%)$ casi siempre o siempre perciben apoyo, después de la intervención de enfermería $74(49,4 \%)$ con frecuencia sienten apoyo de amigos, vecinos y compañeros; 27 (18,1\%) a veces perciben apoyo, $28(18,6 \%)$ casi siempre o siempre y $21(14 \%)$ casi nunca lo perciben (tabla 2$)$.

\begin{tabular}{|c|c|c|c|c|}
\hline \multirow[b]{2}{*}{$\begin{array}{l}\text { FAMILIAR Y OTROS } \\
\text { SIGNIFICATIVOS }\end{array}$} & \multicolumn{2}{|c|}{ Antes } & \multicolumn{2}{|c|}{ Después } \\
\hline & $\mathbf{N}^{\bullet}$ & $\%$ & $\mathbf{N}^{\circ}$ & $\%$ \\
\hline Casi nunca & 27 & 17,9 & 17 & 11,4 \\
\hline$A$ veces & 38 & 25,4 & 22 & 14,5 \\
\hline Con frecuencia & 65 & 43,3 & 77 & 51,4 \\
\hline Casi Siempre o & 20 & 13,4 & 34 & 22,7 \\
\hline \multicolumn{5}{|l|}{ Siempre } \\
\hline Total & 150 & 100 & 150 & 100 \\
\hline \multicolumn{5}{|l|}{$\begin{array}{l}\text { AMIGOS, VECINOS O } \\
\text { COMPAÑEROS DE } \\
\text { TRABAJO }\end{array}$} \\
\hline Casi nunca & 23 & 15,3 & 21 & 14 \\
\hline$A$ veces & 48 & 32,1 & 28 & 18,6 \\
\hline Con frecuencia & 63 & 41,9 & 74 & 49,4 \\
\hline Casi Siempre o & 16 & 10,6 & 27 & 18,1 \\
\hline \multicolumn{5}{|l|}{ Siempre } \\
\hline Total & 150 & 100 & 150 & 100 \\
\hline
\end{tabular}

El índice de Barthel para la evaluación de la capacidad funcional en la alimentación y actividades motoras antes de la intervención de enfermería que el 123 (82\%) son independientes para su alimentación, el $27(18 \%)$ necesita ayuda, después de la intervención de enfermería el 150 (100\%) adultos mayores son independientes para alimentarse. En las actividades motoras de trasladarse de la cama a la butaca o a la silla de ruedas antes de la intervención de enfermería 139 $(92,7 \%)$ son independientes y $11(7,3 \%)$ necesitan ayuda, después de la intervención de enfermería, 140 (93,3\%) son independientes, 10 (6,6\%) necesitan ayuda para dicho traslado. En deambular: antes de la intervención de enfermería que 134 (89,3\%) son independientes para deambular, el 8,7\% (13) necesitan ayuda y $3(2 \%)$ son dependientes, después de la intervención de enfermería el $146 \quad(97,3 \%)$ son independientes, $3(2 \%)$ necesitan ayuda y $1(0,7 \%)$ es dependiente para deambular. Subir escaleras: antes de la intervención de Enfermería el $80(53,3 \%)$ son independientes para subir y bajar escaleras, $56(37,3 \%)$ necesitan ayuda y 14 $(9,3 \%)$ son dependientes, después de la intervención de enfermería $83(55,3 \%)$ son independientes, 58 (38,7\%) necesitan ayuda y $9(6 \%)$ es dependiente para subir y bajar escaleras (tabla 3 ).

TABLA 3 INDICE DE BARTHEL PARA LA EVALUACIÓN DE LA CAPACIDAD FUNCIONAL EN LA ALIMENTACIÓN Y
ACTIVIDADES MOTOPAS

\begin{tabular}{|c|c|c|c|c|}
\hline \multirow[t]{2}{*}{ CAPACIDAD FUNCIONAL } & \multicolumn{2}{|c|}{ Antes } & \multicolumn{2}{|c|}{ Después } \\
\hline & $\mathbf{N}^{\circ}$ & $\%$ & $\mathbf{N}^{\circ}$ & $\%$ \\
\hline \multicolumn{5}{|l|}{ ALIMENTACIÓN } \\
\hline Independiente & 123 & 82 & 150 & 100 \\
\hline Necesita ayuda & 27 & 18 & 0 & $\mathbf{0}$ \\
\hline Dependiente & 0 & 0 & 0 & 0 \\
\hline Total & 150 & 100 & 150 & 100 \\
\hline \multicolumn{5}{|l|}{$\begin{array}{l}\text { TRASLADARSE DE } \\
\text { LA CAMA A LA } \\
\text { BUTACA O LA } \\
\text { SILLA DE RUEDAS }\end{array}$} \\
\hline Independiente & 139 & 92,7 & 140 & 93,3 \\
\hline Necesita ayuda & 11 & 7,3 & 10 & 6,6 \\
\hline Dependiente & 0 & 0 & 0 & 0 \\
\hline Total & 150 & 100 & 150 & 100 \\
\hline \multicolumn{5}{|l|}{ DEAMBULAR } \\
\hline Independiente & 134 & 89,3 & 146 & 97,3 \\
\hline Necesita ayuda & 13 & 8,7 & 3 & 2 \\
\hline Dependiente & 3 & 2 & 1 & 0,7 \\
\hline Total & 150 & 100 & 150 & 100 \\
\hline \multicolumn{5}{|l|}{$\begin{array}{l}\text { SUBIR Y BAJAR } \\
\text { ESCALERAS }\end{array}$} \\
\hline Independiente & 80 & 53,3 & 83 & 55,3 \\
\hline Necesita ayuda & 56 & 37,3 & 58 & 38,7 \\
\hline Dependiente & 14 & 9,3 & 9 & 6 \\
\hline Total & 150 & 100 & 150 & 100 \\
\hline
\end{tabular}

El índice de Barthel para la evaluación de hábitos fisiológicos antes de la intervención de enfermería En la micción (el control de orina) $107(71,3 \%)$ son independientes o continente, durante al menos 7 días, 42 (28\%) necesitan ayuda accidente excepcional (máximo uno/24 horas) y $1(0,7 \%)$ es incontinente o con colocación de sonda vesical incapaz de cambiarse la bolsa, después de la intervención de enfermería 140 (93,3\%) son independientes o continente, $9(6 \%)$ necesitan ayuda y 1 $(0,7 \%)$ es dependientes para la micción. En la utilización de retrete o inodoro 126 (84\%) son independientes (entrar y salir, limpiarse y vestirse), $22(14,7 \%)$ necesitan alguna ayuda, pero puede hacer algo sólo y $2(1,3 \%)$ son dependiente, después de la intervención de enfermería 143 (95,3\%) son independientes, 6 (4\%) necesitan ayuda y $1(0,7 \%)$ es dependientes. En control de heces antes de la intervención de enfermería $113(75,3 \%)$ son independiente (continente), necesita ayuda 36 (24\%): accidente excepcional (uno/semana) y $1(0,7 \%)$ dependiente: incontinente (o necesita que le suministren enema), después de la intervención de enfermería $131(87,3 \%)$ son independiente (continente), necesita ayuda 
18 (12\%): accidente excepcional (uno/semana) y $1(0,7 \%)$ dependiente: incontinente (o necesita que le suministren enema) (tabla 4).

\begin{tabular}{|c|c|c|c|c|}
\hline & & & & \\
\hline & $\mathbf{N}^{\circ}$ & $\%$ & $\mathbf{N}^{\circ}$ & $\%$ \\
\hline \multicolumn{5}{|l|}{ MICcIóN } \\
\hline Independiente & 107 & 71,3 & 140 & 93,3 \\
\hline Necesita ayuda & 42 & 28 & 9 & 6 \\
\hline Dependiente & 1 & 0,7 & 1 & 0,7 \\
\hline Total & 150 & 100 & 150 & 100 \\
\hline \multicolumn{5}{|l|}{$\begin{array}{l}\text { UTILIZACIÓN DEL RETRETE } \\
\text { O INODORO }\end{array}$} \\
\hline $\begin{array}{l}\text { Independiente (entrar y salir, } \\
\text { limpiarse y vestirse) }\end{array}$ & 126 & 84 & 143 & 95,3 \\
\hline $\begin{array}{l}\text { Necesita alguna ayuda o } \\
\text { puede hacer algo solo }\end{array}$ & 22 & 14,7 & 6 & 4 \\
\hline Dependiente & 2 & 1,3 & 1 & 0,7 \\
\hline Total & 150 & 100 & 150 & 100 \\
\hline \multicolumn{5}{|l|}{ CONTROL DE HECES } \\
\hline $\begin{array}{l}\text { Independiente } \\
\text { (continente) }\end{array}$ & 113 & 75,3 & 131 & 87,3 \\
\hline $\begin{array}{l}\text { Necesita ayuda } \\
\text { (accidente excepcional } \\
\text { (uno/semana) }\end{array}$ & 36 & 24 & 18 & 12 \\
\hline $\begin{array}{l}\text { Dependiente } \\
\text { (incontinente (o necesita que } \\
\text { le suministren enema) }\end{array}$ & 1 & 0,7 & 1 & 0,7 \\
\hline Total & 150 & 100 & 150 & 100 \\
\hline
\end{tabular}

Fuente: Índice de Barthel

El índice de Barthel para la evaluación de la higiene personal en bañarse/ducharse antes de la intervención de enfermería $118(78.7 \%)$ necesitan ayuda, $6(17,3 \%)$ son independientes y $26(4 \%)$ son dependientes, después de la intervención de enfermería 119 (79,3\%) necesitan ayuda para bañarse/ducharse, 18 (12\%) dependientes, $13 \quad(8,7)$ independientes. Vestirse y desvestirse $100(66,7 \%), 45(30 \%)$ necesitan ayuda, dependiente $5(3,3)$ y después de la intervención de enfermería $136(90,7 \%), 8$ (12\%) son dependientes y $13(8,7 \%)$ son independientes. En vestirse y desvestirse: antes de la intervención de enfermería 136 $(90,7 \%)$ son independientes incluyendo botones, cremalleras, cordones, etc, 12 (8\%) necesitan ayuda y $2(1,3 \%)$ son dependientes. Arreglarse: antes de la intervención de enfermería $112(74,7 \%)$ necesitan ayuda, dependiente 26 $(17,3 \%)$ e independientes $12(8 \%)$, después de la intervención enfermería 131 (87,3\%) necesitan ayuda, 13 (8,7\%) son independientes y $6(4 \%)$ son dependientes para lavarse la cara, las manos, peinarse, afeitarse, maquillarse, etc. (tabla 5).

TABLA 5

ÍNDICE DE BARTHEL PARA LA EVALUACIÓN DE LA HIGIENE PERSONAL

\begin{tabular}{|c|c|c|c|c|}
\hline & \multicolumn{2}{|c|}{ Antes } & \multicolumn{2}{|c|}{ Después } \\
\hline & $\mathbf{N}^{\circ}$ & $\%$ & $\mathbf{N}^{\circ}$ & $\%$ \\
\hline \multicolumn{5}{|l|}{$\begin{array}{l}\text { BAÑARSE O } \\
\text { DUCHARSE }\end{array}$} \\
\hline Independiente & 6 & 17,3 & 13 & 8,7 \\
\hline Necesita ayuda & 118 & 78,7 & 119 & 79,3 \\
\hline Dependiente & 26 & 4 & 18 & 12 \\
\hline Total & 150 & 100 & 150 & 100 \\
\hline \multicolumn{5}{|l|}{$\begin{array}{l}\text { VESTIRSE Y } \\
\text { DESVESTIRSE }\end{array}$} \\
\hline Independiente & 100 & 66,7 & 136 & 90,7 \\
\hline Necesita ayuda & 45 & 30 & 12 & 8 \\
\hline Dependiente & 5 & 3,3 & 2 & 1,3 \\
\hline Total & 150 & 100 & 150 & 100 \\
\hline \multicolumn{5}{|l|}{ ARREGLARSE } \\
\hline Independiente & 12 & 8 & 13 & 8,7 \\
\hline Necesita ayuda & 112 & 74,7 & 131 & 87,3 \\
\hline Dependiente & 26 & 17,3 & 6 & 4 \\
\hline Total & 150 & 100 & 150 & 100 \\
\hline
\end{tabular}

En la evaluación del estado cognitivo de los adultos mayores antes de la intervención de enfermería 55 (36,6\%) tienen un deterioro cognitivo, $52(34,7 \%)$ se encuentran borderline y 43 $(28,7 \%)$ se encuentran normales, después de la intervención de enfermería $61(40,6 \%)$ se encuentran borderline, 46 $(30,7 \%)$ normales y $43(28,7 \%)$ son adultos mayores con deterioro cognitivo (tabla 6). 
TABLA 6

EVALUACIÓN DEL ESTADO COGNITIVO

\begin{tabular}{lcccc}
\hline & \multicolumn{2}{c}{ Antes } & \multicolumn{2}{c}{ Después } \\
\hline PUNTAJE FINAL & $\mathrm{N}^{\circ}$ & $\%$ & $\mathrm{~N}^{\circ}$ & $\%$ \\
$\begin{array}{l}\text { 0-23 Deterioro cognitivo } \\
\text { (<65 años) }\end{array}$ & 55 & 36,6 & 43 & 28,7 \\
$\begin{array}{l}24-29 \text { Borderline } \\
\text { (>65 años) }\end{array}$ & 52 & 34,7 & 61 & 40,6 \\
$\begin{array}{l}30-34 \text { Normal } \\
\text { (>65 años) }\end{array}$ & 43 & 28,7 & 46 & 30,7 \\
Total & 150 & 100 & 150 & 100 \\
\hline
\end{tabular}

Fuente: Test de Minimental

La evaluación de la intervención de Enfermería se realizó por medio de tres instrumentos: la escala de Zimet para valorar la percepción del apoyo social antes de la intervención una puntuación de 37,081 y después 42,190 con una diferencia significativa $p<0,001$. La escala de Barthel para valorar la independencia del paciente para realizar las actividades básicas de la vida diaria antes de la intervención una puntuación de 55,855 y después de la intervención 94,219 con una diferencia significativa $p<0,001$. el test de Minimental para valorar el estado cognitivo antes de la intervención una puntuación de 29,138 y después de la intervención 32,021 con una diferencia significativa $p<0,001$ (tabla 7).

TABLA 7

EVALUACIÓN DE LA INTERVENCIÓN DE ENFERMERÍA POR LA ESCALA DE ZIMET, ÍNDICE DE BARTHEL Y EL TEST MINIMENTAL

\begin{tabular}{|c|c|}
\hline \multicolumn{2}{|c|}{ ESCALA DE ZIMET } \\
\hline & Total \\
\hline ZIMET ANTES & 37,081 \\
\hline ZIMET DESPUÉS & 42,190 \\
\hline \multicolumn{2}{|c|}{ ÍNDICE DE BARTHEL } \\
\hline \multicolumn{2}{|r|}{ Total } \\
\hline BARTHEL ANTES & 55,855 \\
\hline BARTHEL DESPUÉS & 94,219 \\
\hline \multicolumn{2}{|c|}{ TEST DE MINIMENTAL } \\
\hline & Total \\
\hline MINIMENTAL ANTES & 29,138 \\
\hline MINIMENTAL DESPUÉS & 32,021 \\
\hline
\end{tabular}

*T de Student $p<0,05$.

\section{DISCUSIÓN.}

En la investigación actual las características demográficas en el grupo de adultos mayores el rango de edad de 60-79 años, predominando de 60-64 años y el género masculino, a diferencia de Guerrero y Yépez, evidenciaron una edad mínima de 60 años y máxima de 101 años, un mayor número de edad 60-69 años en el sexo femenino (34) y SeguraCardona y col., igualmente de sexo femenino, la edad promedio de 71,1 $\pm 8,21$ años, el rango de edad 60-112 años, el $68,6 \%$ de los adultos mayores de 60-74 años, denominados adultos mayores jóvenes, el 29,2 \% entre los 75-89 años, considerados adultos mayores viejos, el 1,9\% de ellos tenían entre 90-99 años, denominados adultos mayores longevos y el $0,2 \%$ presentaron una edad mayor de 100 años (35).
En relación al apoyo social percibido por los adultos mayores en el presente trabajo con la intervención de enfermería se incrementó la percepción de apoyo de familiares, amigos, vecinos o compañeros, similar a Fernández y Manrique que resaltaron el apoyo social percibido como un componente cognitivo de la ayuda recibida, de la interacción directa con la red social, donde el personal de enfermería genera cambios en la conducta individual y las actitudes en la interacción entre el adulto mayor y uno o más miembros de su familia o amigos, creando una red social responsable, capaz de proporcionar al adulto mayor: satisfacción de las necesidades emocionales, participación activa en la vida comunitaria, fomento de conductas saludables y una mayor calidad de vida, se le debe demostrar que alguien está disponible, a través de la presencia física para dar muestras de afecto, de cariño, mediante abrazos, invitaciones a caminar, a pasear, el envío de tarjetas, 
de flores, la compañía en las oraciones y el escuchar atentamente; se agregan otras maneras alternativas de ofrecer apoyo como es el uso del internet, las llamadas telefónicas, que disminuyen la incertidumbre, la ansiedad, el aislamiento y la depresión de los adultos mayores (36).

También Zapata-López y col., establecen que el apoyo social percibido es un componente importante, surge de la necesidad del adulto mayor en tener la capacidad para insertarse e integrarse a un grupo social, ratificando la necesidad en geriatría de comunicarse con los semejantes (37), Zurita y col., el envejecimiento exitoso, implica que el individuo sea capaz de integrarse y desenvolverse de manera eficaz tanto en su familia como en la sociedad, superando las barreras que le impidan desarrollar todo su potencial y mantener un nivel adecuado de bienestar personal (38), además el Comité de Cuidado Familiar para Adultos Mayores establece que el factor de apoyo de la familia será siempre la mejor garantía para el bienestar físico y espiritual de las personas mayores (39). En la investigación de Araya y col., el apoyo social medido por MSPSS reportó un promedio de pre-intervención 34,029 y post-intervención 37,286 con cambios positivos en los adultos mayores en la percepción de apoyo social, debido a las actividades con fuerte componente grupal, participativo, social con la reconexión con las redes sociales disponibles en la comunidad, apoyando la percepción de apoyo social, pero las mediciones de funcionalidad no demostraron tener cambios estadísticamente significativos (40).

La evaluación de la capacidad funcional de los adultos mayores a través del índice de Barthel en la investigación actual, después de las intervenciones de enfermería establecen que son independientes para su alimentación, trasladarse de la cama a la butaca o a la silla de ruedas antes, para deambular, subir y bajar escaleras, utilización de retrete o inodoro, en el control de orina o cambiarse la bolsa de la sonda vesical y en el control de las heces similar a Hernández y col., donde los adultos mayores tiene altos índices de independencia como arreglarse (92.9\%), comer y bañarse $(91.8 \%)$, orinar y cuidar de la sonda si cuenta con ella $(90.8 \%)$, deambular, usar el sanitario y defecar (89.9\%), trasladarse de forma independiente de un sofá a la cama $(86.7 \%)$, subir y bajar escaleras (83.2\%) (41). Las intervenciones de enfermería para fortalecer la capacidad funcional de los adultos mayores están fundamentadas en las siguientes modelos teóricos: de Virginia Henderson que sustenta la importancia de aplicar estrategias de Enfermería basadas en satisfacer las necesidades básicas, ya que el rol del enfermero es complementar la autonomía de la persona, para lograr la independencia, desarrollando un modelo basado en las 14 necesidades, brindando una atención integral partiendo desde una valoración exhaustiva de problemas y formulación de diagnósticos de enfermería para posteriormente planificar, ejecutar y evaluar cuidados encaminadas a satisfacer las necesidades de los adultos mayores, fomentando su recuperación y nivel de independencia (42), También en la teoría de autocuidado de Dorothea Orem es indispensable para que los adultos mayores adquieran hábitos para el mejoramiento de su estado de salud, a través de la práctica de autocuidado en nutrición, vestimenta, higiene, autoprotección, recreación, actividad física y el uso adecuado de dispositivos de ayuda, que fomentan la independencia del adulto mayor (43). Para fomentar el autocuidado el modelo de Pender integra la enfermería a la ciencia del comportamiento, identificando los factores que influyen en los comportamientos saludables, es decir, una guía para explorar la motivación o desmotivación de los ancianos al adquirir comportamientos de autocuidado para un envejecimiento saludable (44).
En trabajo actual la evaluación del estado cognitivo de los adultos mayores después de la intervención de enfermería se evidenció disminución del deterioro cognitivo e incremento de la evaluación borderline y normal, similar a Calatayuda y col., realizaron un programa de estimulación para prevenir el deterioro de las capacidades cognitivas en una población de adultos mayores con cognición normal, por medio de cuadernos de colores de activación mental, que fortalecieron las áreas de memoria orientación, lenguaje, práctica, gnosis cálculo, percepción, razonamiento lógico, atenciónconcentración y programación, con efectos beneficiosos a nivel de cognición global en dominios altamente relevantes, para prevenir el deterioro cognitivo como la orientación temporal y la praxis, medido con el test mini-mental a los 6 meses y al año después de la intervención, con relevancia clínica para controlar el deterioro cognitivo leve desde Atención Primaria y promover la salud cognitiva de los adultos mayores (45), a diferencia de Camargo-Hernández y Laguado-Jaimes, donde el deterioro cognitivo es una situación frecuente en la población adulta mayor institucionalizada, ya que el $41,7 \%$ tienen deterioro cognitivo grave, estableciendo la necesidad de intervenciones integrales, donde el profesional de enfermería cumpla un papel importante en la conservación de la capacidad de la memoria de esta población a lo largo del tiempo $(46,47)$

Díaz y col; evidenciaron que el $23 \%$ de los adultos mayores de 65 años prevalecía la demencia como enfermedad neurológica, lo cual es un signo de alarma en el área de salud pública y que refleja la importancia que tiene el direccionar los cuidados de enfermería con intervenciones como entrenamiento de la memoria, estimulación cognitiva, facilitar el aprendizaje, potencialización de la disposición de aprendizaje, restructuración cognitiva, terapia de reminiscencia y orientación a la realidad en el adulto mayor, para prevenir alteraciones del área cognitiva en este grupo poblacional.

\section{CONCLUSIONES:}

En geriatría las intervenciones en Enfermería permiten la prevención, el diagnóstico precoz de enfermedades crónicas y promover el aprendizaje de conductas para fortalecer la capacidad funcional de los adultos mayores, a través del autocuidado, las características demográficas en el grupo de adultos mayores el rango de edad de 60-79 años, predominando de 60-64 años y el género masculino, en relación al apoyo social percibido por los adultos mayores en el presente trabajo con la intervención de enfermería se incrementó la percepción de apoyo de familiares, amigos, vecinos o compañeros.

La evaluación de la capacidad funcional de los adultos mayores a través del índice de Barthel, después de las intervenciones de enfermería establecen que son independientes para su alimentación, trasladarse de la cama a la butaca o a la silla de ruedas antes, para deambular, subir y bajar escaleras, utilización de retrete o inodoro, en el control de orina o cambiarse la bolsa de la sonda vesical y en el control de las heces. El estado cognitivo de los adultos mayores después de la intervención de enfermería se evidenció disminución del deterioro cognitivo e incremento de la evaluación borderline y normal.

FINANCIAMIENTO: no hubo fuentes de financiamiento.

CONFLICTOS DE INTERESES: Los autores declaran no tener conflictos de interés. 


\section{REFERENCIAS BIBLIOGRÁFICAS:}

1. Orem, D. Modelos y teorías de enfermería. Barcelona: Harcourt Brace. Orem, D. E. Conceptos de enfermería en la práctica, México: Ediciones Científicas y Técnicas, S.A. 1999.

Disponible en: file://C:/Users/HP\%20STORE/Downloads/Dialnet-TeoriaDelDeficitDeAutocuidado-2534034\%20(1).pdf.

2. Organización de las Naciones Unidas. Envejecimiento. 2017. Disponible en: http://www.un.org/es/sections/issuesdepth/ageing/index.html.

3. Rodríguez B, Moreno J. Efecto de estrategias de cuidado de enfermería en el adulto mayor con deterioro de la movilidad física y conductas generadoras de la salud en centros de bienestar en la ciudad de Bucaramanga. Rev Cuid. 2011;2(2):155-160.

Disponible en: https://dialnet.unirioja.es/servlet/articulo?codigo $=4563108$

4. Asociación Americana de Facultades de Enfermería, Fundación John A. Hartford Instituto para Enfermería Geriátrica. New York University College of Nursing Adultos mayores: Competencias Recomendadas y Guía Curricular para el cuidado de enfermería geriátrico en el Programa de Licenciatura (Baccalaureate). New York. 2005.

Disponible en: http://www.sld.cu/galerias/pdf/sitios/gericuba/competencias recomendadas y guia curricular.pdf.

5. Arnadottir, SA, Bruce, J., Lall, R., Withers, EJ, Underwood, M., Shaw, F., Sheridan, R., Hossain, A., Lamb, SE y Grupo de estudio Pre-FIT. La importancia de diferentes dominios de fragilidad en una muestra basada en la población en Inglaterra. BMC geriatrics. 2020;20(1):16. https://doi.org/10.1186/s12877-019-1411-9.

6. Checa-López M, Oviedo-Briones M, Pardo-Gómez A, Gonzales-Turín J, Guevara-Guevara T, Carnicero JA, Alamo-Ascencio S, Landi F, Cesari M, Grodzicki T, Rodriguez-Mañas L; FRAILTOOLS study protocol: a comprehensive validation of frailty assessment tools to screen and diagnose frailty in different clinical and social settings and to provide instruments for integrated care in older adults. BMC Geriatr. 18 de marzo de 2019; 19 (1): 86. doi: 10.1186 / s12877-019-1042-1.

7. World economic and social survey. development in an ageing world. New York: United Nations, 2007. Disponible en: https://www.google.com/search?rlz=1C1SQJL esEC792EC792\&ei=aXDsXN7jKeKxggfsqYbgBg\&q=biocombustibles\&oq=bioco mbus\&gs I=psy-ab.1.0.0l10.6907.11681..13641..0.0..0.295.2517.2-9.....0...1..gws-wiz....0..0i71j0i67j0i131i67.REoxW57uVcM 8. $\quad$ Flores Ramírez M., Arriaga Bueno R., Silos Vaquera A., Perez Requejo K. Percepción del Proceso de Envejecimiento en Adultos Mayores en México. 2018;27(1):1-8.

Disponible en: https://revistas.unlp.edu.ar/escenarios/article/view/8088/7005.

9. Leiton Espinoza, Z.E. El envejecimiento saludable y el bienestar: un desafío y una oportunidad para enfermería. Rev. Enfermería Universitaria. 2016; 13 (3): 139-141.

Disponible en: http://revista-enfermeria.unam.mx/ojs/index.php/enfermeriauniversitaria/article/view/76

10. Organización Mundial de la Salud OMS. Informe mundial sobre el envejecimiento y la salud. WHO/FWC/ALC/15.01. 2015.

Disponible en: http://apps.who.int/iris/bitstream/handle/10665/186471/WHO FWC ALC 15.01 spa.pdf.

11. Instituto Nacional de Estadística y Censos (INEC). Difusión de Resultados Definitivos del censo Nacional de Vivienda. 2010. Provincia de Manabí. Disponible en: http://www.ecuadorencifras.gob.ec/base-de-datos-censo-de-poblacion-y-vivienda/.

12 Bohórquez V.; Castañeda B.; Trigos L.; Orozco L.; Camargo A. Eficacia de la intervención enfermera para cuidadores con cansancio del rol del cuidador. Revista Cubana de Enfermería. 2011;27(3):210-219. Disponible en: http://scielo.sld.cu/scielo.php?script=sci arttext\&pid=S0864-03192011000300005

13. Lloyd-Sherlock P, McKee M, Ebrahim S, Gorman M, Greengross S, Prince M et al. Población, envejecimiento y salud . The Lancet . Abril de 2012; 379 (9823): 1295-1296. https://doi.org/10.1016/S0140-6736(12)60519-4.

14. Smith A. Grand challenges of our aging society: workshop summary. Washington (DC): National Academies Press. 2010. Disponible en: http://www.nap.edu/catalog/12852/grand-challenges-of-our-agingsociety-workshop.

15. Organización Panamericana de la Salud. Enseñanza de la Enfermería en Salud del Adulto Mayor. Washington. 2012. DC. OPS: Serie Recursos Humanos para la Salud $N^{\circ}$ 59.isbn: 978-92-75-31671-9 Disponible en: file:///C:/Users/HP\%20STORE/Downloads/nursing-salud-adulto-mayor-2012-spa.pdf.

16. Puello-Alcocer EC, Amador-Ahumada C, Ortega-Montes JE. Impacto de las acciones de un programa de enfermería con enfoque promocional y de autocuidado en la capacidad funcional de adultos mayores. Rev Univ. Salud. 2017;19(2):152-162. DOI: http://dx.doi.org/10.22267/rus.171902.78

17. Bulechek G, Butcher H, Dochterman J, Wagner Ch. Clasificación de Intervenciones de Enfermería (NIC) Ed. Elsevier 6 edición. 2013.

Disponible en: https://bibliosjd.org/2020/04/02/nnueva-edicion-nic-noc/\#.X JegtgzblU

18. Urquiza, M., Fernandez, N., Arrinda, I., Sierra, I., Irazusta, J., \& Rodriguez Larrad, A. Nutritional Status Is Associated with Function, Physical Performance and Falls in Older Adults Admitted to Geriatric Rehabilitation: A Retrospective Cohort Study. Nutrients 2020; 12(9):2855. https://doi.org/10.3390/nu12092855.

19. Chisag GM. Atención de enfermería en el adulto mayor. Enferm Inv.2018; 3(Sup.1):59-61

Disponible en: http://www.sld.cu/galerias/pdf/sitios/pdvedado/adulto mayor.pdf.

20. Hui Lin Ong, ,Janhavi Ajit Vaingankar, Edimansyah Abdin ,Rajeswari Sambasivam ,Restria Fauziana ,Min-En Tan ,Siow Ann Chong ,Richard Roshan Goveas ,Peak Chiang Chiam yMythily Subramaniam. Resiliencia y sobrecarga en cuidadores de adultos mayores: efectos moderadores y mediadores del apoyo social percibido. Psiquiatría BMC 2018; 18: (27). https://doi.org/10.1186/s12888-018-1616-z

21. Ortiz Parada, Manuel; Baeza Rivera, María José. Propiedades psicométricas de una escala para medir apoyo social percibido en pacientes chilenos con diabetes tipo 2. Univ. Psychol. 2011;10(1):189-196.

Disponible en: http://www.scielo.org.co/scielo.php?script=sci abstract\&pid=S1657-92672011000100016\&lng=es

22. Navarro-Loli, J. S., Merino-Soto, C., Dominguez-Lara, S., \& Lourenço, A. Estructura interna de la Multidimensional Scale of Perceived Social Support (MSPSS) en adolescentes peruanos. Revista Argentina de Ciencias del Comportamiento, 2019;11(1):38-47.

Disponible en: https://revistas.psi.unc.edu.ar/index.php/racc/article/view/21532. 
23. Arechavala MC, Miranda C. Validación de una escala de apoyo social percibido en un grupo de adultos mayors adscritos a un programa de hipertensión de la región metropolitana. Cienc enferm. 2002;8(1):49-55. Disponible en: https://scielo.conicyt.cl/scielo.php?script=sci arttext\&pid=S0717-95532002000100007.

24. Hui Lin Ong, ,Janhavi Ajit Vaingankar,Edimansyah Abdin ,Rajeswari Sambasivam ,Restria Fauziana ,Min-En Tan ,Siow Ann Chong ,Richard Roshan Goveas ,Peak Chiang Chiam yMythily Subramaniam. Resiliencia y sobrecarga en cuidadores de adultos mayores: efectos moderadores y mediadores del apoyo social percibido. Psiquiatría BMC 2018;18(27). https://doi.org/10.1186/s12888-018-1616-z

25. Centro Nacional de Programas Preventivos y Control de Enfermedades, Secretaría de Salud de México. Principios del abordaje gerontológico en la persona adulta mayor e intervenciones básicas. 2012. Primera edición. Disponible en: www.salud.gob.mx $<$ www.cenavece.salud.gob.mx

26. Rojas-Gualdrón, D.F., Segura C., A., Cardona A., D., Segura C., A., \& Garzón D. M.O. Análisis Rasch del Mini Mental State Examination (MMSE) en adultos mayores de Antioquia, Colombia. Rev. CES Psico 2017;10(2):17-27.

Disponible en: http://www.scielo.org.co/pdf/cesp/v10n2/2011-3080-cesp-10-02-00017.pdf

27. Intervenciones de Enfermería para el Cuidado del Adulto Mayor con Deterioro Cognoscitivo. México: Instituto Mexicano del Seguro Social; 2015:(38)

Disponible en: www.imss.gob.mx

28. Organización Mundial de la Salud OMS. Envejecimiento y salud. La salud de las personas de edad. Serie Informes técnicos número 779. 2017. Ginebra: Disponible en: www.who.int/mediacentre/factsheets/fs404/es.

29. Stanik-Hutt J, Newhouse RP, White KM. The Quality and Effectiveness of Care Provided by Nurse Practitioners. J Nurse Pract. 2013;9(8):492-500. Disponible en: https://www.ncbi.nlm.nih.gov/books/NBK169424/

30. Vivaldi, F.; Barra, E. Bienestar Psicológico, Apoyo Social Percibido y Percepción de Salud en Adultos Mayores. Ter Psicol. 2012;30(2):23-29. Disponible en: https://scielo.conicyt.cl/pdf/terpsicol/v30n2/art02.pdf

31. Araya Alejandra X., Herrera María Soledad, Iriarte Evelyn, Rioja Romina. Evaluación de la funcionalidad y fragilidad de las personas mayores asistentes a centros de día. Rev Med Chile 2018;146(8):864-871.

Disponible en: https://biblat.unam.mx/es/revista/revista-medica-de-chile/articulo/evaluacion-de-la-funcionalidad-y-fragilidad-delas-personas-mayores-asistentes-a-centros-de-dia.

32. Gavilanes V., Mendoza E., Fernández G. Rol de la enfermería en el bienestar físico del adulto mayor. Enferm Inv. 2019 4(5):24-28.

Disponible en:https://revistas.uta.edu.ec/erevista/index.php/enfi/article/view/727

33. Afonso, Rosa Marina; Duarte, Elina; Pereira, Henrique; da Graça Esgalhado, Maria. Construcción de un programa individual de reminiscencia para mayores con demencia residentes en una institución International. Journal of Developmental and Educational Psychology. 2010; 2(1):643-650.

Disponible en: https://www.redalyc.org/pdf/3498/349832325067.pdf

34. Guerrero N, Yépez MC. Factores asociados a la vulnerabilidad del adulto mayor con alteraciones de salud. Rev Univ. salud. 2015;17(1):121-131. Disponible en: http://www.scielo.org.co/pdf/reus/v17n1/v17n1a11.pdf.

35. Segura-Cardona Alejandra, Cardona-Arango Doris, Segura-Cardona Ángela y Garzón-Duque María. Riesgo de depresión y factores asociados en adultos mayores. Antioquia, Colombia. 2012. Rev. salud pública. 2015; 17(2):184-194. DOI: http://dx.doi.org/10.15446/rsap.v17n2.41295.

36. Fernández Alba R., Manrique-Abril F.G.Rol de la enfermería en el apoyo social del adulto mayor. Enferm. glob. 2010;(19):19. Disponible en:

http://scielo.isciii.es/scielo.php?script=sci arttext\&pid=S1695-61412010000200016\&lng=es

37. Zapata-López, Bertha I., Delgado-Villamizar, Norma L., Cardona-Arango, Doris Apoyo social y familiar al adulto mayor del área urbana en Angelópolis, Colombia 2011. Revista de Salud Pública. 2015, 17 (6), 848-860.

Disponible en: https://www.redalyc.org/articulo.oa?id=42244105003

38. Zurita Pinto D., Gordillo Alarcón S., Rosero Alan R., Reyes Zamora J., Maldonado Landázuri M. Actividad diaria según índice de Barthel en adultos mayores, Ibarra. Maldonado LMV/et al/Enfermería Investiga, Investigación, Vinculación, Docencia y Gestión. 2016;1(3):112-116.

Disponible en: https://dialnet.unirioja.es/servlet/revista?codigo=25734.

39. Committee on Family Caregiving for Older Adults; Board on Health Care Services; Health and Medicine Division; National Academies of Sciences, Engineering, and Medicine; Schulz R, Eden J, editors. Families Caring for an Aging America. Washington (DC): National Academies Press (US); 2016 Nov 8. 3, Family Caregiving Roles and Impacts

Disponible en: https://www.ncbi.nlm.nih.gov/books/NBK396409/

40. Cecilia Pinto S., Roxana Lara J., Elena Espinoza L., Pamela Montoya C. Propiedades psicométricas de la escala de apoyo social percibido de Zimet En Personas Mayores De Atención Primaria de Salud. 2013.

Disponible en: http://scielo.isciii.es/scielo.php?script=sci arttext\&pid=S1132-12962014000100018

41. Hernández Ramírez Magdalena, Lumbreras Delgado Irma, Hernández Vicente Irma Alejandra, Báez Alvarado María del Rocío, Juárez Flores Clara Arlina, Banderas Tarabay José Antonio, Banda González Olga Lidia. Valoración de la funcionalidad y dependencia del adulto mayor en áreas rurales. Revista Médica de la Universidad Veracruzana. 2016;16(2):7-24.

Disponible en: https://www.medigraphic.com/pdfs/veracruzana/muv-2016/muv162a.pdf

42. Cabascango Allauca Lourdes Maribel, Hinojosa Chariguamán Ana Gabriela, Remache Agualongo Lourdes Morayma, Olalla García María Humbelina. Aplicación del proceso de atención de enfermería basado en el modelo teórico de Virginia Henderson, en el centro gereatrico "Amawta Wasi Samay" Guaranda-Bolívar. Ciencia digital. 2019;3(1):54-63.

Disponible en: https://cienciadigital.org/revistacienciadigital2/index.php/CienciaDigital/article/view/250

44. Aristizábal Hoyos Gladis Patricia, Blanco Borjas Dolly Marlene, Sánchez Ramos Araceli, Ostiguín Meléndez Rosa María. El modelo de promoción de la salud de Nola Pender: Una reflexión en torno a su comprensión. Enferm. Univ. 2011;8(4):16-23.

Disponible en: http://www.scielo.org.mx/scielo.php?script=sci arttext\&pid=S1665-70632011000400003\&lng=es.

45. Calatayud E, Plo F, Muro C. Análisis del efecto de un programa de estimulación cognitiva en personas con envejecimiento normal en Atención Primaria: ensayo clínico aleatorizado [Analysis of the effect of a program of cognitive stimulation in elderly people with normal aging in primary care: Randomized clinical trial]. Aten Primaria. 2020 Jan;52(1):38-46. Spanish. doi: 10.1016/j.aprim.2018.09.007. Epub 2018 Nov 20. PMID: 30470457; PMCID: PMC6939008. 
46. Camargo-Hernández K del C, Laguado-Jaimes E. Grado de deterioro cognitivo de los adultos mayores institucionalizados en dos hogares para ancianos del área metropolitana de Bucaramanga - Santander, Colombia. Rev Univ. Salud. 2017;19(2):163170. DOI: http://dx.doi.org/10.22267/rus.171902.79

47. Díaz R, Marulanda F, Martínez MH. Prevalencia de deterioro cognitivo y demencia en mayores de 65 años en una población urbana colombiana. Rev Acta Neurológica Colomb. 2013;29(3):141-151.

Disponible en: https://www.scielosp.org/article/rcsp/2018.v44n3/e979/ 